\title{
Chaotic neuron clock
}

\author{
A. Bershadskii ${ }^{1,2}$ and Y. Ikegaya ${ }^{3,4}$ \\ ${ }^{1}$ ICAR - P.O. Box 31155, Jerusalem 91000, Israel and ${ }^{2}$ ICTP - Strada Costiera 11, \\ I-34100 Trieste, Italy \\ ${ }^{3}$ Laboratory of Chemical Pharmacology, Graduate School of Pharmaceutical Sciences, \\ University of Tokyo, Tokyo 113-0033, and ${ }^{4}$ Precursory Research for Embryonic Science \\ and Technology, Japan Science and Technology Agency, Kawaguchi 332-0012, Japan
}

\begin{abstract}
A chaotic model of spontaneous (without external stimulus) neuron firing has been analyzed by mapping the irregular spiking time-series into telegraph signals. In this model the fundamental frequency of chaotic Rössler attractor provides (with a period doubling) the strong periodic component of the generated irregular signal. The exponentially decaying broad-band part of the spectrum of the Rössler attractor has been transformed by the threshold firing mechanism into a scaling tale. These results are compared with irregular spiking time-series obtained in vitro from a spontaneous activity of hippocampal (CA3) singular neurons (rat's brain slice culture). The comparison shows good agreement between the model and experimentally obtained spectra.
\end{abstract}

Keywords: Chaos, neuron, spikes, spontaneous activity

\section{Introduction}

In order to work together the brain neurons have to make an adjustment of their rhythms. The main problem for this adjustment is the very noisy environment of the brain neurons. For pure periodic inner clocks this adjustment would be impossible due to the noise. Nature, however, has another option. This option is a chaotic clock. In chaotic attractors certain characteristic frequencies can be embedded by broad-band spectra, that makes them much more stable to the noise perturbations [1]. We will present empirical evidence supporting the proposition that the Rössler chaotic attractor in combination with an appropriate threshold passage mechanism could be 


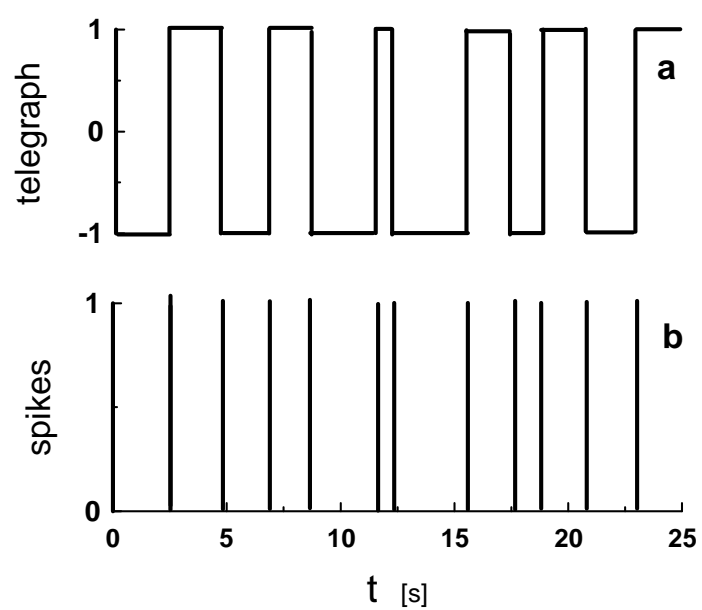

Figure 1: Mapping of a spike train (figure 1b) into a telegraph signal (figure 1a).

used as a simple model of the spontaneous (without external stimulus) firing activity frequently observed in in vitro hippocampal neurons [2], [3] .

The spontaneous firing activity should be more simple and self-consistent than a reaction of a neuron to the external stimuli. This can allow an analysis of the inner neuron clock in its "free-run" mode. Consideration of the most frequently firing neurons can also help in this direction. Besides its pure academic significance for studying the neurons' firing [4] the spontaneous activity provides a considerable contribution to network development [5], [6], information processing [7]-[10], and behavioral responses [11],[12]. It is known that periodic spontaneous bursts of the activity can convey information about sensory stimuli [13], [14]. Spontaneous activity in brain slice preparations purely reflects the intrinsic properties of local circuits and individual neurons and hence allows for the investigation of the internal dynamics of neuronal networks [15]-18].

All types of information, which is received by sensory system, are encoded by nerve cells into sequences of pulses of similar shape (spikes) before they are transmitted to the brain. Brain neurons use such sequences as main instrument for intercells connection (both for tuning of their chaotic clocks and information-wise). The information is reflected in the time intervals between successive firings (interspike intervals of the action potential train, see Fig. 1b [19]). There need be no loss of information in principle when converting from dynamical amplitude information to spike trains [20] and the irregular spike sequences are the foundation of neural information processing. Although understanding of the origin of interspike intervals irregularity 


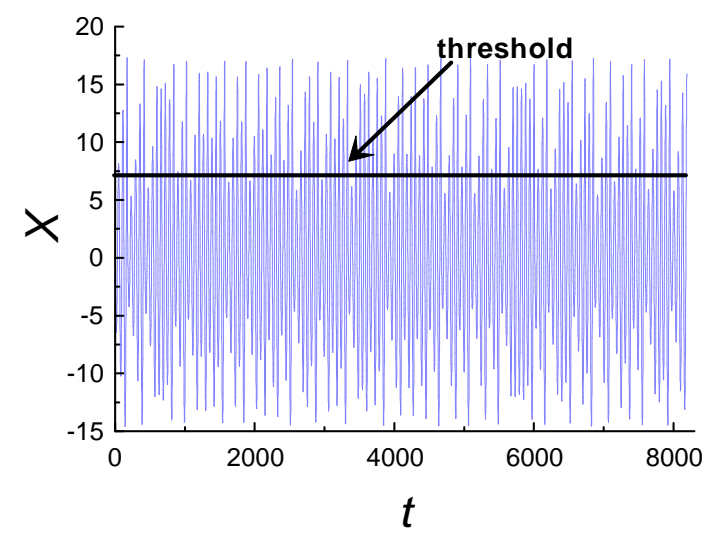

Figure 2: X-component fluctuations of a chaotic solution of the Rössler system Eq. (1) $(a=0.15, b=0.20, c=10.0)$.

has important implications for elucidating the temporal components of the neuronal code and for treatment of such mental disorders as depression and schizophrenia, the problem is still very far from its solution (see, for instance, Ref. [21] and references therein). Motivation to study the hippocampus in relation to depression is based on the recently discovered evidences of its deep involvement in this mental disorder. The hippocampus is a significant part of a brain system responsible for behavioral inhibition and attention, spatial memory, and navigation. It is also well known that spatial memory and navigation of the rats is closely related to the rhythms of their moving activity. On the other hand, the hippocampus of a human who has suffered long-term clinical depression can be as much as $20 \%$ smaller than the hippocampus of someone who has never been depressed [22]. One can speculate that in the case of depression the chaotic neuron clocks can be broken in a significant part of the brain neurons. That can result in certain decoherence in different parts of the brain.

Treatment of the spiky signals by analytical methods also presents a difficult problem. In order to apply the Fourier transform method one can use different mappings: mapping of the finite spikes into Dirac delta functions or into telegraph signals, for instance (cf. Ref. [23]). For a single neuron firing the amplitude of the spikes are almost identical to each other and the neural information is coded in the length of the interspike intervals and their positions on the time axis [19], [20], therefore it is the most direct way to map the spike train into a telegraph time signal, which has values -1 from one side of a spike and values +1 from another side of the spike with a chosen timescale resolution. An example of such mapping is given in figure 1. While the 


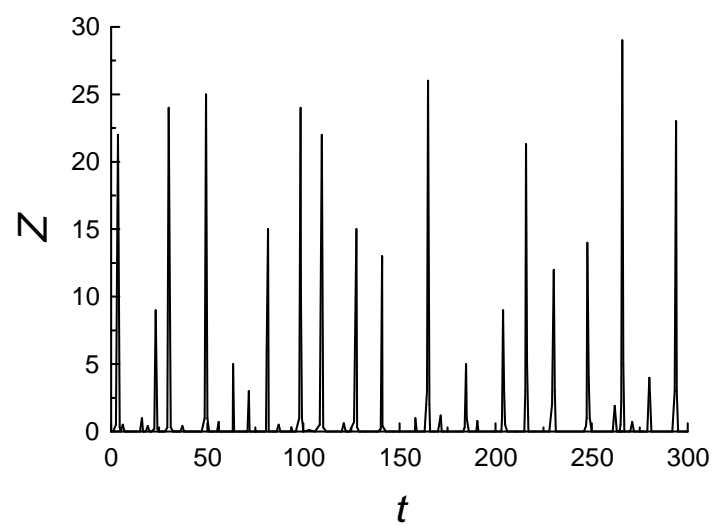

Figure 3: Z-component fluctuations of a chaotic solution of the Rössler system (the parameters are the same as in Fig. 2).

information coding is here the same as for the corresponding spike train, the Fourier transform methods are quite applicable to analysis of the telegraph time-series [24].

\section{A spontaneous firing model: the Rössler system}

Nerve cells are surrounded by a membrane that allows some ions to pass through while it blocks the passage of other ions. When a neuron is not sending a signal it is said to be "at rest". At rest neurons exhibit very small conductance of sodium ions and slightly larger potassium conductance against a high concentration of intracellular potassium ions. The resting value of membrane electrochemical potential - $P$ (the voltage difference across the neural membrane) of a neuron is about $-70 \mathrm{mV}$. If some event (a stimulus) causes the resting potential to move toward $0 \mathrm{mV}$ and the depolarization reaches about $-55 \mathrm{mV}$ (a "normal" threshold) a neuron will fire an action potential. The action potential is an explosive release of charge across plasma membrane and its surrounding that is created by a depolarizing current. If the neuron does not reach this critical threshold level, then no action potential will fire. Also, when the threshold level is reached, an action potential of a fixed size will always fire (for any given neuron the size of the action potential is always the same).

Recent reconstructions of a driver of the membrane potential using the neuron spike trains indicate the Rössler oscillator as the most probable (and simple) candidate (see, for instance, Refs. 25]-[31]). Figure 2 shows as example the x-component fluctuations of a chaotic solution of the Rössler system [32. 


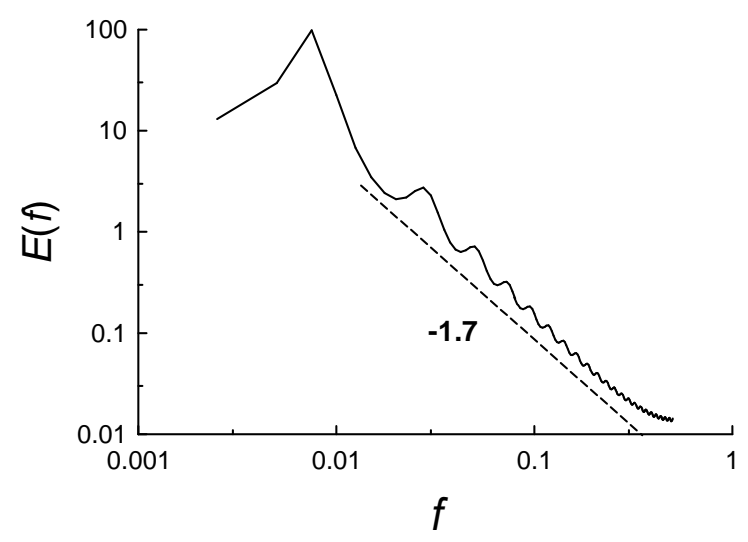

Figure 4: Spectrum of the telegraph signal corresponding to the spike train generated by the $\mathrm{x}$-component fluctuations overcoming the threshold $x=7$. The dashed straight line indicates a power law Eq. (3) in the log-log scales.

$$
\frac{d x}{d t}=-(y+z) ; \quad \frac{d y}{d t}=x+a y ; \quad \frac{d z}{d t}=b+x z-c z
$$

where $\mathrm{a}, \mathrm{b}$ and $\mathrm{c}$ are parameters. At certain values of the parameters $\mathrm{a}, \mathrm{b}$ and $\mathrm{c}$ the $z$-component of the Rössler system is a spiky time series Fig. 3 (see also Refs. [33], 34]).

It can be shown that the Rössler system and the well known HindmarshRose model [35] of neurons are subsystems of the same differential model with a spiky component [34]. Previously the 'spiky' component of such models was interpreted and studied as a simulation of a neuronal output. For the spontaneous neuron firing (without external stimulus), however, we suggest to reverse the approach and consider the spiky variable as the main component of the electrical input (which naturally should have a 'spiky' character, see above) to the neuron under consideration. For any given neuron the height of the spikes, which the neuron generates, is about the same. However, the heights of the spikes generated by different neurons are different. Also the signals coming from different neurons to the neuron under consideration have to go through the electrochemical passes with different properties. Therefore, the spiky z-time-series (Fig. 3) can naturally represent a multineuron signal, which can be considered as a spontaneous input for the neuron under consideration. If we use the usual interpretation of the $x$-component as a driver of the membrane potential $P(x)$ and the $y$-component as that taking into account the transport of ions across the membrane through the ion channels [35], then addition of the spiky z-component (representing in present 


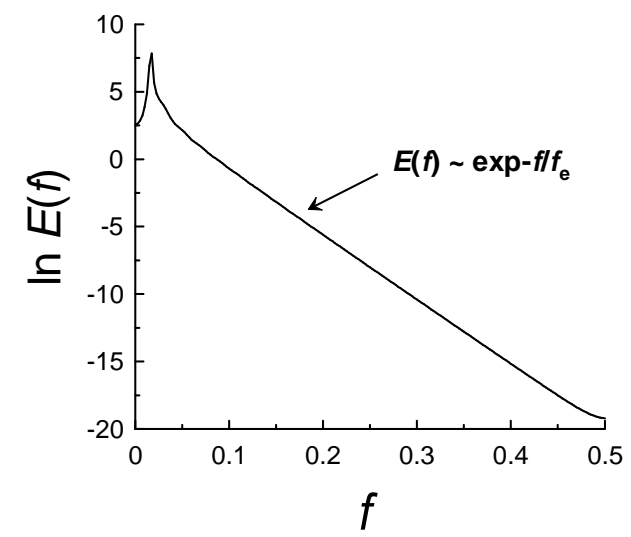

Figure 5: Spectrum of the x-component fluctuations shown in Fig. 2. We used the semi-log axes in order to indicate exponential decay of the spectrum.

model the multineuron spontaneous input) in the dynamical equation for $\mathrm{x}$ component is similar to the addition of an external input-component to the dynamical x-equation in the Hindmarsh-Rose model. Then, the quadratic nonlinearity in the third equation of the system Eq. (1) can be interpreted as a simple (in the Taylor expansion terms) feedback of the neuron to the main component of the neuronal input. This model with the strong nonlinear feedback can be relevant to the most active neurons of a spontaneously active brain (see below results of an in vitro experiment with a spontaneous brain activity). The details of the function $P(x)$ is not significant for the threshold firing process, what really matters is that the membrane potential function $P(x)$ reaches its firing value when (and only when) its argument $x$ crosses certain threshold from below. In this simple model the driving variable $x$ may overcome its threshold value (Fig. 2) due to the deterministic (chaotic) spontaneous stimulus. Let us consider an output spike signal resulting from overcoming a threshold value $x=7$, for instance. Fig. 4 shows spectrum of the telegraph signal corresponding to the spike train. In order to understand what is going on here we show in figure 5 spectrum of the x-component itself. The semi-log scales are used in these figures in order to indicate exponential decay in the spectra (in the semi-log scales this decay corresponds to a straight line):

$$
E(f) \sim e^{-f / f_{e}}
$$

While the low-frequency peak in the spectrum corresponds to the fundamen- 


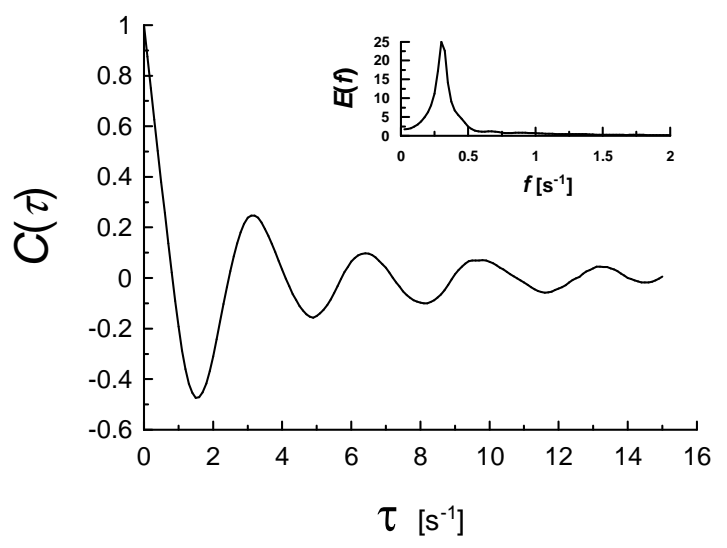

Figure 6: Autocorrelation function for the telegraph signal corresponding to the cell-21 (800 spikes). The insert shows corresponding spectrum.

tal frequency, $f_{0}$, of the Rössler chaotic attractor, the rate of the exponentional decay (the slope of the straight line in Fig. 5) provides us with and additional characteristic frequency $f_{e}$ Eq. (2). It should be noted that for a wide class of deterministic systems a broad-band spectrum with exponential decay is a generic feature of their chaotic solutions Refs. [36]-[39].

Thus Rössler chaotic attractor has two clocks: fundamental with frequency $f_{0}$ and decaying with frequency $f_{e}$. If one compares Fig. 4 and Fig. 5 one can see that the fundamental clock survives the threshold crossing (with a period doubling, see also Fig. 9). The decaying clock, however, does not survive the threshold crossing: the exponential decay in Fig. 5 has been transformed into a scaling (power law) decay in Fig. 4

$$
E(f) \sim f^{-\alpha}
$$

(with $\alpha \simeq 1.7$ ), which has no characteristic frequency (scale invariance).

In the above presented model the fundamental frequency of chaotic Rössler attractor provides (with a period doubling) the strong periodic component of the generated irregular signal. This periodic component can be utilized by Nature as a chaotic clock of the spontaneous neuron firing (see next section). The exponentially decaying broad-band part of the spectrum of the Rössler attractor has been transformed by the threshold firing mechanism into a scaling tale. To understand mechanism of this transformation is rather difficult problem since the mechanism of generation of the exponential spectrum by the chaotic systems is itself an unsolved problem (see, for instance, Refs. 


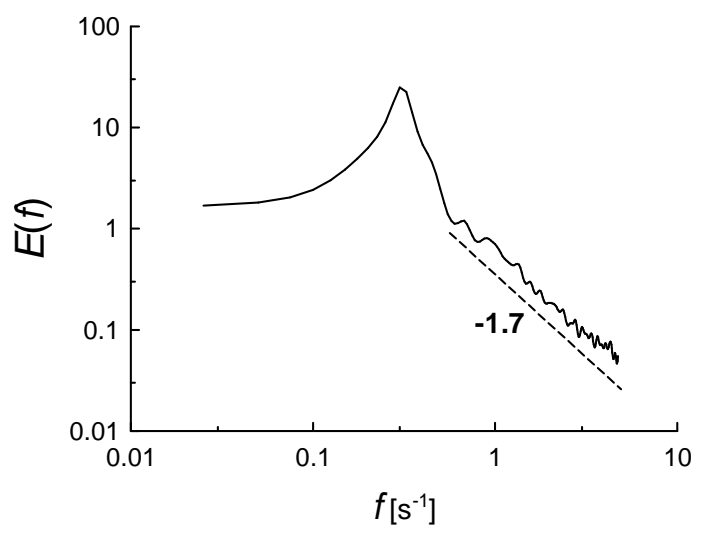

Figure 7: Spectrum of the telegraph signal corresponding to the cell-21 (800 spikes) in $\log -\log$ scales. The dashed straight line indicates a power law Eq. 4: $E(f) \sim f^{-1.7}$.

[36]-[39]). The scaling exponent value ' $-1.7^{\prime}$ ' is not sensitive to a reasonable variation of the threshold value $(\sim 20 \%)$ and even to Gaussian fluctuations of the threshold value. Therefore, it is not just a coincidence that the scaling law in the Rössler case agrees with results of the in vitro experiment reported in the next section (cf. also [24],[40]). The power-law spectrum Eq. (3) with the exponent $\alpha \simeq 1.7$ is an indication of a strongly non-ergodic system, with perennial aging, or maybe that an infinitely aged correlation function is predominant due to noise or truncations. Moreover, the in vitro data, which we used for comparison (see next Section) seem to fit a renewal hypothesis, compatible with this scaling exponent, and the theory of a recent Ref. [41] (see also Ref. [42]).

\section{In vitro spontaneous brain activity}

In order to compare this simple model consideration with the experimental data we have analyzed spike trains obtained in vitro from a spontaneous activity in CA3 hippocampal slice culture of a Wistar/ST rat (the raw data and the detail description of the experiment can be found online at http://hippocampus.jp/data and in Refs. [2],[3]), In the in vitro experiment a functional imaging technique with multicell loading of the calcium fluorophore was used in order to obtain the spike trains of spontaneously active singular neurons (hippocampal pyramidal cells) in the absence of external input. In this experiment different levels of activity were observed for different neurons [2], [3]. We take for our analysis the two most active neurons (http://hippocampus.jp/data- Data-006, cell-21, with 800 spikes in the 


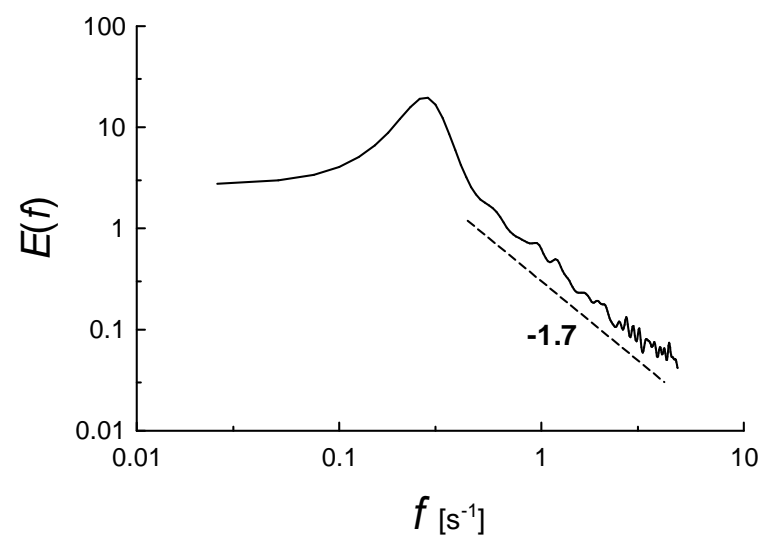

Figure 8: As in Fig. 7 but for cell-25 (D-006, 692 spikes).

time-series; and a cell-25, with 692 spikes). The spike trains were mapped to telegraph signals as it is described above. Figure 6 shows the autocorrelation function for the telegraph signal corresponding to the cell-21 (800 spikes). Insert in the Fig. 6 shows the corresponding spectrum. Both the correlation function and the spectrum provide a clear indication of a strong periodic component in the irregular signal (the oscillations in the correlation function and the peak in the spectrum). The periodic component can be seen at frequency $f_{0} \simeq 0.3 \mathrm{~Hz}$. Figure 7 shows the spectrum in log-log scales. One can see that at high frequencies the spectrum exhibits a scaling behavior Eq. (3) (power law: $E(f) \sim f^{-1.7}$, as indicated by the dashed straight line). The real power law can be more pronounced but under the experimental conditions individual spikes emitted at firing rates higher than $5 \mathrm{~Hz}$ were experimentally inseparable [2],[3]. Figure 8 shows spectrum of the telegraph signals corresponding to the spike train obtained for the cell-25 (D-006, 692 spikes). The spectrum is rather similar to the spectrum shown in Fig. 7 ( for cell-21). The more broad peak in Fig. 8 can be related to the poorer statistics for the cell-25 (number of spikes 692) in comparison with cell-21 (number of spikes 800). One can compare Figs. 7 and 8 with Fig. 4 to see very good reproduction of the main spectral properties. Figure 9 shows also a superposition of the autocorrelation functions for the telegraph signals corresponding to the cell-21 (the solid line) and to the spike train generated by the the Rössler attractor fluctuations overcoming the threshold $x=7$ (circles). In order to make the autocorrelation functions comparable a rescaling has been made 


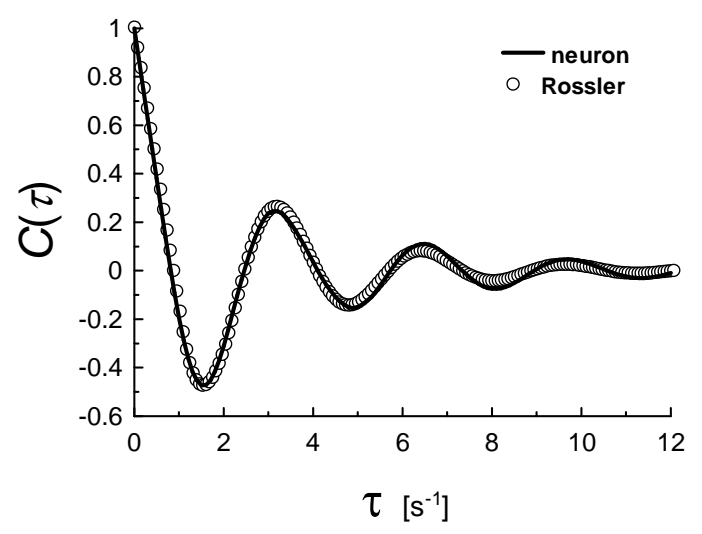

Figure 9: Autocorrelation functions for the telegraph signals corresponding to the cell21 and to the spike train generated by the Rössler attractor fluctuations overcoming the threshold $x=7$ (circles). In order to make the autocorrelation functions comparable a rescaling has been made for the Rössler autocorrelation function.

for the Rössler autocorrelation function: the scaling coefficient is equal to 0.024 (the time step size for the Rössler solution was 0.01). As in the case of the spectra the comparison of the autocorrelation functions is not sensitive to a reasonable variation of the threshold value ( $20 \%$ ) and even to Gaussian fluctuations of the threshold value.

\section{Conclusion}

Simple Rössler system has properties, which allow to use it as an adequate model of spontaneous neuron activity (that includes a multineuron input represented by the spiky z-component of the Rössler attractor, Fig. 3, and a strong nonlinear feedback). A mapping of the spike time series into telegraph signals (that preserves completely intact the 'frequency modulated' information of the spike series) allows to compare spectral properties of the model threshold firing (Fig. 4) with analogous firing of the in vitro brain neurons spontaneous activity (Figs. 7 and 8). This investigation can be considered as an additional confirmation and specification of the already existing evidences (Refs. [25]-[31]) that the Rössler system can be a driver of spontaneous neuron firing. On the other hand, it is known that the spontaneous firing of brain neurons accounts for about $80 \%$ of the metabolic energy consumed by the brain [43]. Therefore the spontaneous neural activity should have a very significant neurobiological function (cf. Introduction).

Spontaneous neural activity with multineuron nonlinear interactions has 
been studied mainly in neuronal networks (see, for instance, Refs. [2]-[18]). In present simple model a multineuron input and a strong nonlinear feedback are simulated in the frames of a single self-consistent low-dimensional system with a chaotic solution. On the other hand, we do not know any study where a fully chaotic system could make anomalous behaviors emerge, so it seems that studying threshold passage is enough to have that effect.

Of course, in vivo neuron signals can be much more complex. The relatively simple firing of the in vitro spontaneously active hippocampal neurons can be used in order to reveal the underlying neuron dynamics.

\section{Acknowledgments}

We thank Takahashi N., Sasaki T., Usami A., and Matsuki N. for help in obtaining the data. We also thank Greenberg A. for help in computing.

\section{References}

[1] M. I. Rabinovich, and H. D. I. Abarbanel, The role of chaos in neural systems, Neuroscience, 87, (1998) 5-14.

[2] T. Sasaki, N. Matsuki and Y. Ikegaya, Metastability of Active CA3 Networks, J. Neuroscience, 27, (2007) 517-528.

[3] N. Takahashi, T. Sasaki, W. Matsumoto, N. Matsuki, and Y. Ikegaya, Circuit topology for synchronizing neurons in spontaneously active networks, PNAS, 107, (2010) 10244-10249.

[4] A. Mazzoni, F.D. Broccard, E. Garcia-Perez, P. Bonifazi P, et al., (2007) On the Dynamics of the Spontaneous Activity in Neuronal Networks, PLoS ONE, 2 (2007) e439.

[5] A.A. Penn, and C.J. Shatz, Brain waves and brain wiring: the role of endogenous and sensory-driven neural activity in development, Pediatr. Res., 45, (1999) 447-458.

[6] L.I. Zhang, and M.M Poo, Electrical activity and development of neural circuits. Nat. Neurosci., 4, (2001) 1207-1214.

[7] D. Pare, E. Shink, H. Gaudreau, A. Destexhe, E.J. Lang, Impact of spontaneous synaptic activity on the resting properties of cat neocortical pyramidal neurons in vivo. J. Neurophysiol, 79 (1998) 1450-1460. 
[8] J.S. Anderson, I. Lampl, D.C. Gillespie, D. Ferster, The contribution of noise to contrast invariance of orientation tuning in cat visual cortex. Science, 290, (2000) 1968-1972.

[9] C.C. Petersen, T.T. Hahn, M. Mehta, A. Grinvald, and B. Sakmann, Interaction of sensory responses with spontaneous depolarization in layer 2/3 barrel cortex, PNAS, 100, (2003) 13638-13643.

[10] Y. Shu, A. Hasenstaub, M. Badoual, T. Bal, D.A. McCormick, Barrages of synaptic activity control the gain and sensitivity of cortical neurons. J. Neurosci., 23, (2003) 10388-10401.

[11] K.L Briggman, H.D. Abarbanel, Jr. W.B. Kristan, Optical imaging of neuronal populations during decision-making, Science, 307, (2005) 896901.

[12] L.J. Otten, A.H. Quayle, S. Akram, T.A. Ditewig, M.D. Rugg, (2006) Brain activity before an event predicts later recollection, Nat. Neurosci., 9, (2006) 489-491.

[13] T. Kenet, D. Bibitchkov, M. Tsodyks, A. Grinvald, A. Arieli, Spontaneously emerging cortical representations of visual attributes, Nature, 425, (2003) 954-956.

[14] J.N. MacLean, B.O. Watson, G.B. Aaron, R. Yuste, Internal dynamics determine the cortical response to thalamic stimulation, Neuron, 48, (2005) 811-823.

[15] M.V. Sanchez-Vives, and D.A. McCormick, Cellular and network mechanisms of rhythmic recurrent activity in neocortex, Nat. Neurosci., bf 3, (2000) 1027-1034.

[16] J.M. Beggs, and D. Plenz, (2003) Neuronal avalanches in neocortical circuits, J. Neurosci., 23, (2003) 11167-11177.

[17] J.M. Beggs, and D. Plenz, Neuronal avalanches are diverse and precise activity patterns that are stable for many hours in cortical slice cultures, J. Neurosci., 24, (2004) 5216-5229.

[18] C.V. Stewart, and D. Plenz, (2006) Inverted-U profile of dopamineNMDAmediated spontaneous avalanche recurrence in superficial layers of rat prefrontal cortex, J. Neurosci. 26, (2006) 8148-8159. 
[19] F. Rieke, D. Warland, R. de Ruyter van Steveninck, and W. Bialek, Spikes: Exploring the Neural Code (MIT Press, Cambridge, 1997).

[20] T. Sauer, Interspike interval embedding of chaotic signals, Chaos, 5, (1995) 127-133.

[21] C. van Vreeswijk and H. Sompolinsky, Chaos in Neuronal Networks with Balanced Excitatory and Inhibitory Activity, Science, 274 (1996) 17241726.

[22] J.D. Bremner, M. Narayan, E.R Anderson et al., Am. J. Psychiatry, 157, (2000) 115.

[23] S.B. Lowen and M.C. Teich, Fractal renewal processes generate 1/f noise, Phys. Rev. E, 47, (1993) 992-1001.

[24] M. Luković and P. Grigolini, Power spectra for both interrupted and perennial aging processes, J. Chem. Phys. 129, (2008) 184102.

[25] R. Castro and T. Sauer, Chaotic Stochastic Resonance: Noise-Enhanced Reconstruction of Attractors, Phys. Rev. Lett., 79, (1997) 1030-1033 .

[26] R. Castro and T. Sauer, Reconstructing chaotic dynamics through spike filters , Phys. Rev. E, 59, (1999) 29112917.

[27] N. Masuda, and K. Aihara, Bridging Rate Coding and Temporal Spike Coding by Effect of Noise, Phys. Rev. Lett., 88 (2002) 248101.

[28] K. Aihara and I. Tokuda, Possible neural coding with interevent intervals of synchronous firing, Phys. Rev. E, 66, (2002) 026212.

[29] T. Gedeon, M. Holzer and M. Pernarowski, Attractor reconstruction through spike filters and phase locking", Physica D, 178 (2003), 149172 .

[30] N. Crook, W.J. Goh, and M. Hawarat, BioSystems, Pattern recall in networks of chaotic neurons, BioSystems, 87, (2007) 267-274.

[31] T. Pereira, M.S. Baptista, and J. Kurths, Average period and phase of chaotic oscillators, Phys. Lett. A, 362, (2007) 159. 
[32] O.E. Rössler, An equation for continuous chaos, Phys. Lett. A, 57, (1976) 397-398.

[33] C. Lainscsek, C.Letellier and I. Gorodnitsky, Global modeling of the Rössler system from the z-variable, Phys. Lett. A, 314, (2003) 409-427.

[34] C. Lainscsek, I. Gorodnitsky and C. Letellier, Reconstructing dynamics from amplitude measures of spiky time-series, 8th Joint Symposium on Neural Computation, (2001) (available at http://www.its.caltech.edu/ jsnc/2001/Proceedings/).

[35] J.L. Hindmarsh and R.M. Rose, A Model of Neuronal Bursting Using Three Coupled First Order Differential Equations, Proc. R. Soc. Lond. B, 221, (1984) 87-102.

[36] D.E. Sigeti, Survival of deterministic dynamics in the presence of noise and the exponential decay of power spectra at high frequency, Phys. Rev. E, 52, (1995) 2443-2457.

[37] N. Ohtomo, K. Tokiwano, Y. Tanaka, A. Sumi, S.Terachi, and H. Konno, Exponential Characteristics of Power Spectral Densities Caused by Chaotic Phenomena, J. Phys. Soc. Jpn. 64, (1995) 1104-1113.

[38] J. D. Farmer, Chaotic Attractors of an Infinite-Dimensional Dynamical System, Physica D, 4, (1982) 366-393.

[39] U. Frisch and R. Morf, Intermittency in nonlinear dynamics and singularities at complex times, Phys. Rev. A, 23, (1981) 2673-2705 .

[40] P. Grigolini, G. Aquino, M. Bologna, M. Luković, and B. J. West, A theory of $1 / f$ noise in human cognition, Physica A, 388, (2009) 41924204 .

[41] P. Allegrini, D. Menicucci, R. Bedini, A. Gemignani, and P. Paradisi, Phys. Rev. E, 82, (2010) 015103(R).

[42] Personal communication by P. Allegrini.

[43] M. Raichle, The brain dark energy, Science, 314 (2006) 1249-1250. 